\title{
Metastatic Prostate Mass to the Intradural Foramen Magnum Region: A Case Report
}

\author{
Joseph Quillin, B.S. ${ }^{1}$ Prashant Chittiboina, M.D., M.P.H. ${ }^{1} \quad$ Justin Haydel, M.D. ${ }^{1}$ Anil Nanda, M.D., F.A.C.S. ${ }^{1}$ \\ ${ }^{1}$ Department of Neurosurgery, LSU HSC Shreveport, Shreveport, \\ Louisiana \\ J Neurol Surg Rep 2012;73:9-13. \\ address for correspondence and reprint requests Anil Nanda, M.D., \\ F.A.C.S., Chair and Professor, Department of Neurosurgery, P.O. Box \\ 33932, LSU HSC Shreveport, Shreveport, LA 71130-3932 \\ (e-mail: ananda@lsuhsc.edu).
}

\begin{abstract}
Intradural metastatic tumors of the foramen magnum region are extremely rare tumors. We report a 73-year-old patient that presented with right hemiparesis and a recent history of prostate biopsy for an enlarged prostate. Imaging revealed an anterolateral intradural foramen magnum mass with compression of the medulla. A right far lateral approach with condyle preservation was used to resect the mass. Pathological

Keywords

- foramen magnum

- prostate

- metastatic tumor

- intradural

- far lateral examination revealed the tumor as a metastatic prostate mass. The patient had a significant recovery of motor function and was given adjuvant external beam radiation. At the time of last follow-up, the patient had good clinical relief from the preoperative symptoms. To our knowledge, this is the first reported case of an intradural foramen magnum prostate tumor metastasis. We report on multimodal management of this rare, yet morbid presentation of a common tumor.
\end{abstract}

Brain metastases from prostate cancer are relatively rare. There have been previous reports of prostate cancer metastasizing to the skull base; however, the most frequent locations are the sellar and the parasellar regions. Skull base metastases from distant tumors occur in 4 to $9 \%$ of cancer patients. The three most frequent culprits are breast, lung, and prostate cancers, accounting for $\sim 40,14$, and $12 \%$ of cases, respectively. ${ }^{1,2}$ Among these, foramen magnum lesions are even rarer. There exist a few case reports of intradural metastatic lesions to foramen magnum. The reported primary sources for such tumors are melanoma, ${ }^{3}$ pituitary, ${ }^{4}$ and thyroid carcinoma..$^{5}$ To our knowledge, this is the first reported case of a metastatic prostate mass to the intradural foramen magnum region.

\section{Case Report}

This 73-year-old man presented to our institution with a history of generalized weakness in July 2010. He reported having several falls in the previous year and progressive difficulty ambulating over the past month requiring the use of a cane. Neurological examination revealed a right-sided hemiparesis and hyper-reflexia of the upper extremities. Cervical magnetic resonance imaging (MRI) performed at that time revealed contrast-enhancing mass measuring $\sim 2 \times 1.4 \times 1.2 \mathrm{~cm}$ at the level of the foramen magnum. The mass appeared intradural and extramedullary in location and severe displacement of the spinal cord was noted (- Fig. 1). Magnetic resonance angiography performed at that time did not reveal any displacement or invasion of the vertebral artery. Given the location of the mass, the patient was consented for a right-sided far lateral skull base approach for resection.

The patient was placed in a left lateral decubitus position and the head was fixed with Mayfield three-point fixation. The head was positioned such that the saggital sinus was parallel to the floor and the neck was flexed slightly toward the left shoulder. The skin incision was marked $\sim 4 \mathrm{~cm}$ posterior to the posterior pinna and the tip of the mastoid (-Fig. 2d). A 3-inch lazy S-shaped skin incision was made, extending from level of external auditory meatus down to the level of C2-lateral-mass prominence. Following the muscular layer dissection, the vertebral artery was identified at the level of C1. Further exposure was extended to the foramen received

June 30, 2011

accepted after revision

October 5, 2011

published online

February 21, 2012
Copyright (c) 2012 by Thieme Medical Publishers, Inc., 333 Seventh Avenue, New York, NY 10001, USA. Tel: +1(212) 584-4662.
DOI http://dx.doi.org/ 10.1055/s-0032-1301405. ISSN 2193-6358. 

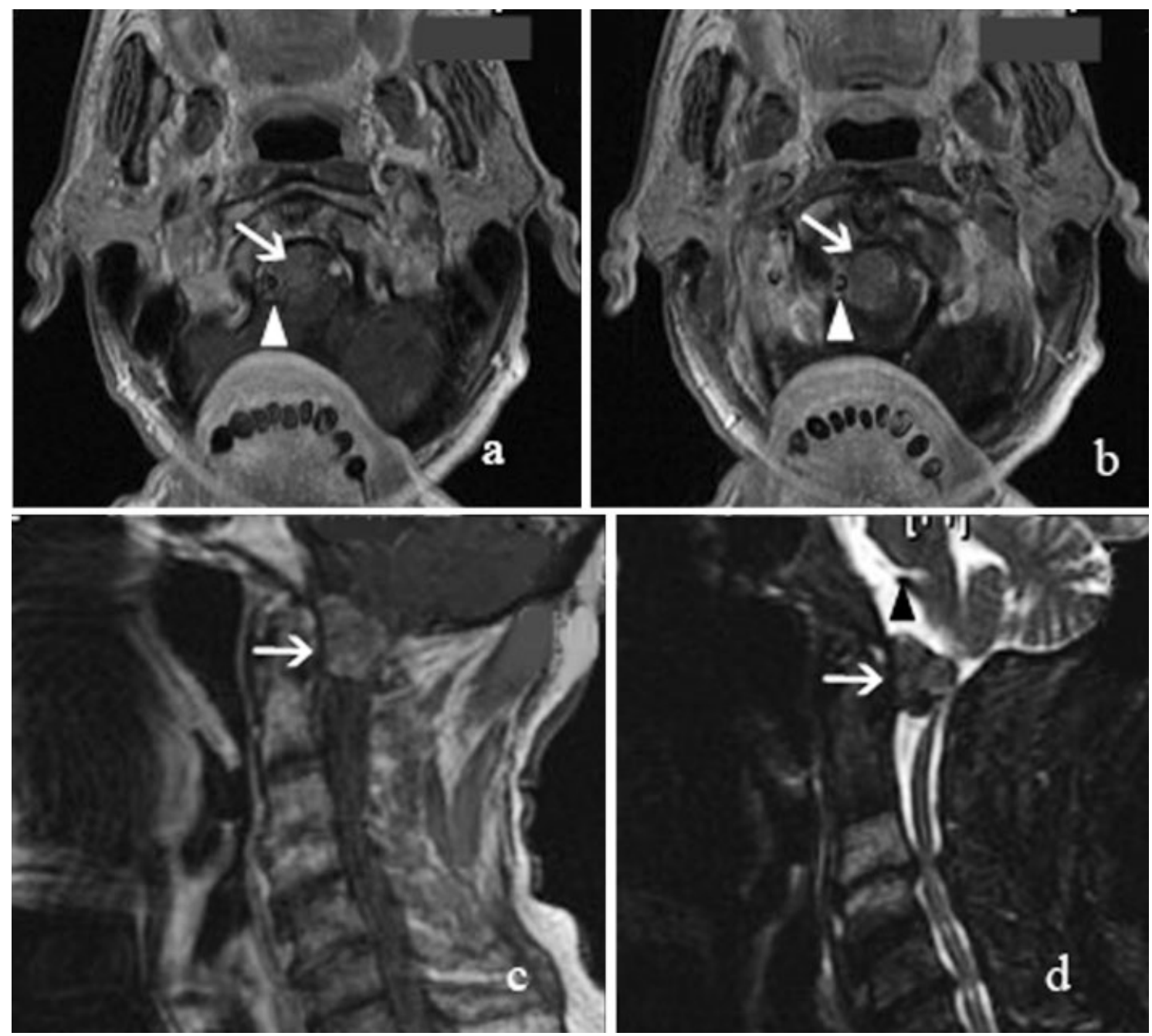

Figure 1 Preoperative magnetic resonance imaging of the craniocervical junction revealing an enhancing mass (a-c) with a significant cord compression and cord edema (d). The white arrows point at the dural base mass arising from the anterolateral dural location at the foramen magnum (a-d). White arrowheads point at the location of laterally displaced right vertebral artery (a, b). A black arrowhead points to the superior displacement of the vertebral artery (d).

magnum and occipital bone. Next, a partial laminectomy of C1 (-Fig. 2c) was performed along with a small occipital craniotomy and the dura was opened in a trap-door fashion. Following this, a soft, hemorrhagic mass was encountered with adhesions to the vertebral artery, posterior inferior cerebellar artery, and the lower cranial nerves ( - Fig. 2a and $\mathbf{2 b}$ ). Following meticulous dissection a gross total resec-

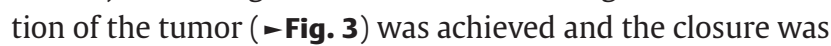
performed in a layered fashion.

After an overnight stay in the neurosurgical intensive care unit, the patient was transferred to the neurosurgical floor. His strength improved gradually with daily physical therapy. Pathology report revealed the mass to be metastatic adenocarcinoma consistent with prostatic origin. The tumor cells were noted to stain positive for prostate specific antigen (PSA). Computed tomography (CT) imaging of the chest abdomen and pelvis with oral and intravenous contrast as well as a bone scan did not reveal any other significant findings. During postoperative hospitalization, a prostate examination revealed the presence of an enlarged prostate.
Additionally, the patient's serum PSA was elevated to $15.9 \mathrm{ng} /$ $\mathrm{mL}$ (reference range 0 to $6.5 \mathrm{ng} / \mathrm{mL}$ ) on the screening test. A diagnostic (quantitative) test revealed an elevated serum PSA at $7.9 \mathrm{ng} / \mathrm{mL}$ (reference range 0 to $6.5 \mathrm{ng} / \mathrm{mL}$ ). Consultations were made to the hematology/oncology, urology, and radiation oncology services. Physical therapy and occupational therapy consults were obtained. The patient was assisted with progressive ambulation and activities of daily living. Approximately 2 weeks later, the patient was discharged to rehabilitation facility in a stable condition with an assessed discharge Karnofsky score of $60 \%$. The discharge plan included adjuvant external beam radiation following staple removal and course of PO flutamide and bicalutamide. He received standard external radiation to the craniocervical junction with a total dose of $3000 \mathrm{cGy}$ in 10 fractions from postoperative days 32 through 46 . He tolerated the radiation well and progressed through physical therapy sessions with increasing bilateral lower and upper extremity strength. During immediate postoperative visits, he was progressively ambulatory. At the time of last follow-up 

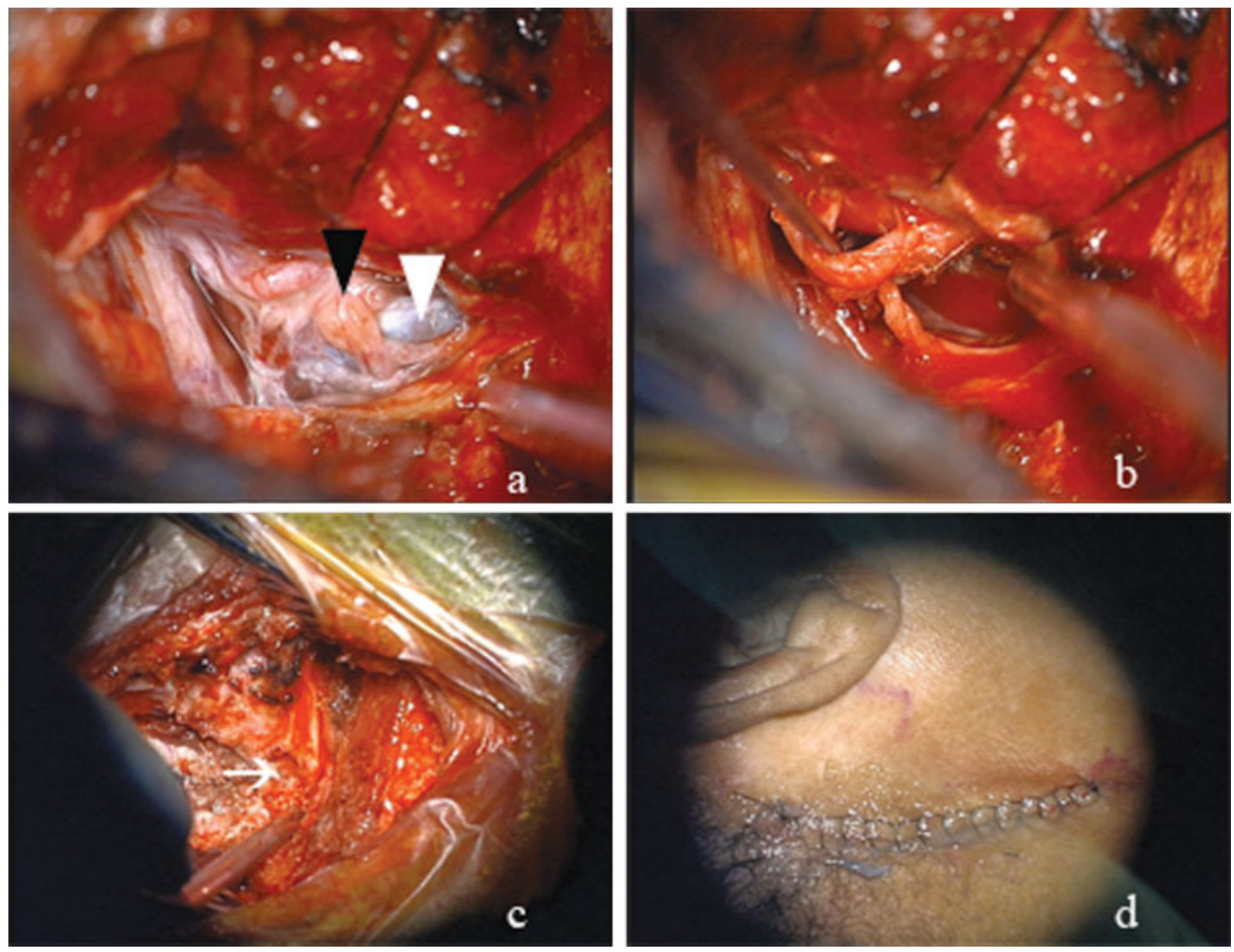

Figure 2 Intraoperative images showing a tumor adherent to the arachnoid and the neurovascular structure at the foramen magnum (a). Hemorrhagic tumor was resected using surgical corridors between the lower cranial nerves (b). Using the minimally invasive far lateral approached described by senior author (A.N.), a small "S"-shaped incision is used to approach the tumors at foramen magnum (d). Additionally, partial unilateral laminectomy of C1 is performed (c). A black arrowhead points at the tumor capsule (a). A white arrowhead points at the lower cranial nerve complex (cranial nerves XI and XII) splayed out over the expansile mass (a). The edge of partially removed C1 lamina is pointed with a white arrow (c).

(9 months postoperative), the patient developed a new onset radiculopathy that was attributed to lumbar stenosis and lateral recess stenosis. Bilateral upper extremity strength remained intact. The patient is continuing with regular follow-ups currently, and no evidence of any bony or softtissue metastasis has been observed in surveillance imaging to date.

\section{Discussion}

Metastatic intradural spinal or cranial prostatic adenocarcinoma is a rare entity. In one large retrospective review of 16,280 patients with metastatic prostate carcinoma, only $0.63 \%$ was found to have metastases to intradural locations in the cranium. The most common sites for metastatic spread were the leptomeninges in $67 \%$, cerebral cortex in $25 \%$, and cerebellum in $8 \%^{6}$. Although intradural metastases from prostate tumors are rare, bony involvement of the skull base has been reported frequently. In a retrospective review of 279 patients in the literature with reported metastases to the skull base between 1963 and 2003, prostatic carcinoma represented $38 \%{ }^{1}$ The clinical presentation of these tumors varies. Some tumors may be clinically silent, while others present with obvious cranial nerve palsies. Greenberg et al identified five syndromes which patients with skull base metastases present with in an attempt to localize lesions more accurately including orbital (7\%), parasellar (16\%), middle fossa (35\%), jugular foramen (16\%), and occipital condyle (21\%). ${ }^{7}$ The mechanism of prostate metastasis is thought to be through the vertebral venous plexus and is frequently closely associated with the dura. ${ }^{8}$ Due to this involvement with the dura, these lesions can be mistaken for subdural hematomas as well as subdural hematomas on radiological evaluation..$^{9-11}$ In our patient, given the gradual onset of symptoms along with the gadolinium-enhanced T1-weighted MRI this lesion was initially felt to represent a meningioma of the foramen magnum. Due to the patient's advanced age, CT imaging with oncology protocol was performed which did not reveal suspicious lesions elsewhere. 

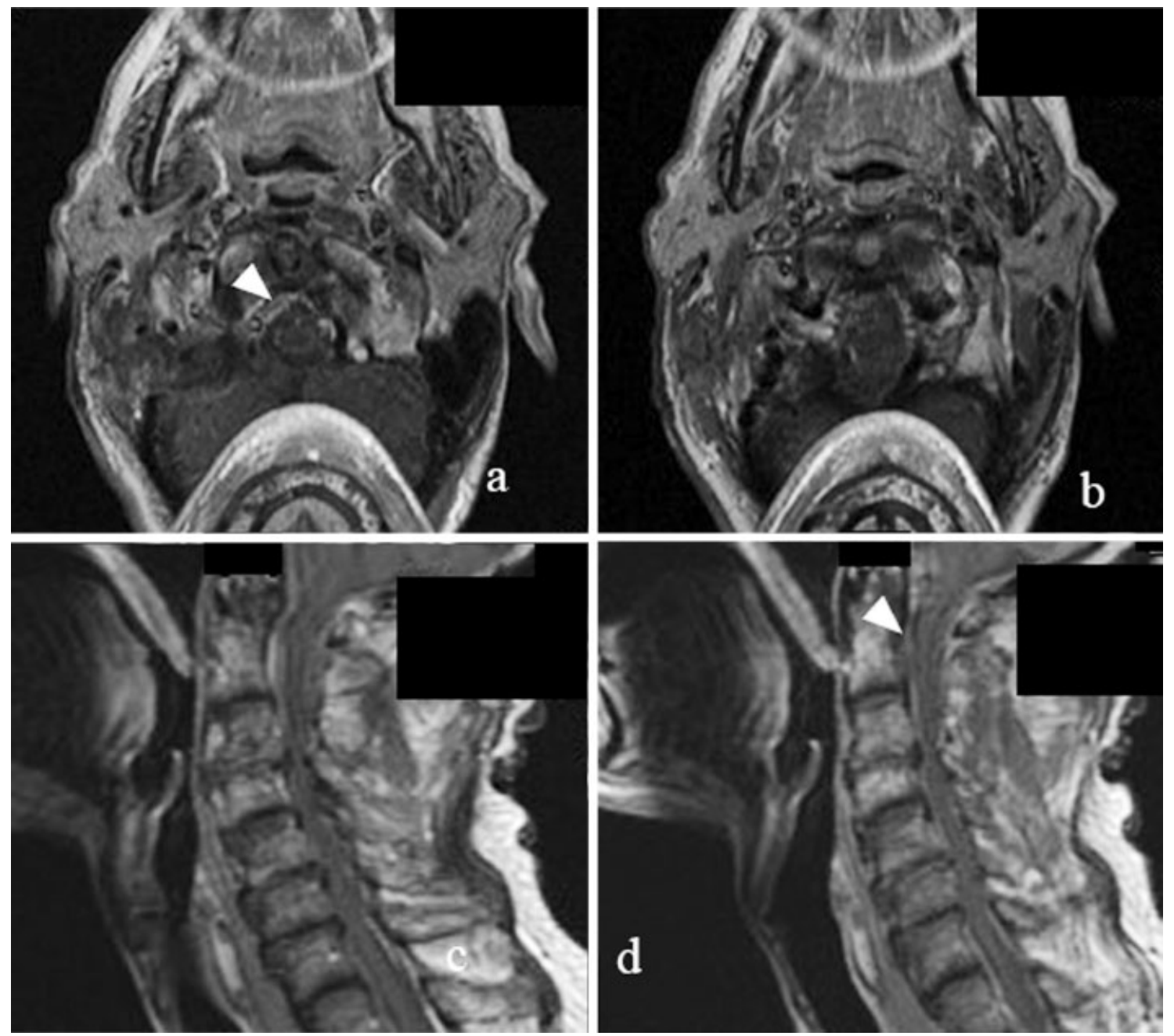

Figure 3 Postoperative magnetic resonance imaging showing a good resection of the foramen magnum mass (a, b). The medulla oblongata and the upper cervical spinal cord do not appear to be under compression (c, d). A white arrowhead points to the dural base of the grossly removed tumor $(a, d)$.

Recently, technical advances have made surgical resection a possible treatment option for lesions of the skull base. Candidates for surgery must be carefully chosen based on their clinical status, functional status, extent of primary disease, and tumor pathology. ${ }^{1}$ In our patient there was no known primary disease. The tumor was extensive in size and causing significant mass effect. Surgery was chosen as the initial therapy for diagnostic as well as therapeutic purposes. We chose a far lateral approach with no condylar resection previously described by our group earlier. ${ }^{12}$

Radiotherapy has long been the primary treatment measure for metastatic skull base lesions. McDermott et al reported in a series of 15 patients with cranial nerve deficits secondary to prostate metastasis that palliative external beam radiation therapy with either $20 \mathrm{~Gy}$ in 5 fractions to the skull base or $30 \mathrm{~Gy}$ in 10 fractions to the whole brain achieved complete response in $67 \%$ of the patients. Although a good resolution of cranial nerve deficits was seen, the length of survival was not similarly affected. Of the 15 patients, 10 patients died within 3 months after receiving radiation therapy and only 3 patients were alive after 1 year following treatment. ${ }^{13}$ O'Sullivan et al reported in a series of 32 patients with cranial nerve deficits due to prostate metastasis that external beam radiation therapy with $20 \mathrm{~Gy}$ in five fractions along with corticosteroids achieved complete response in only $25 \%$ of patients. The median survival following skull base radiotherapy was 3 months. ${ }^{14}$ More recently radiosurgery has been considered an option for skull base metastasis, either as a primary treatment or as a secondary treatment for recurrence. Iwai et al reported 18 patients with metastases to the skull base treated with Gamma Knife radiosurgery (Elekta AB, Stockholm, Sweden). Tumor size in these patients remained stable or decreased in size in 12 (67\%) of subjects; $11(61 \%)$ had complete or partial resolution of symptoms. Mean follow-up was 10.5 months. These lesions must be less than $3 \mathrm{~cm}$ in diameter or less than $10 \mathrm{~cm}^{3}$ in volume. ${ }^{15} \mathrm{In}$ a series of 71 patients, the use of Gamma Knife radiosurgery was shown to be a safe adjuvant to traditional treatment for lesions of the skull base large tumors. The technique consisted of surgical debulking with near total resection followed by Gamma Knife surgery for any residual tumor. This appeared to reduce the potential morbidity in these patients. ${ }^{16}$ 
Multimodality treatment with surgery and radiosurgery is already playing a more prominent role in the management of tumors in difficult-to-reach locations such as the foramen magnum. In our patient, the tumor bed was given external beam radiation in an adjuvant fashion that has resulted in good clinical outcome.

\section{Conclusion}

We present the first reported case of a prostate tumor metastasizing to the foramen magnum. The tumor was surgically removed with a far lateral approach without a condylar resection and followed by adjuvant external beam radiation. We highlight the multimodal management of this rare, yet morbid presentation of a common tumor.

\section{Acknowledgment}

The authors thank Lindsey Hudson for image editing.

\section{References}

1 Laigle-Donadey F, Taillibert S, Martin-Duverneuil N, Hildebrand J, Delattre J-Y. Skull-base metastases. J Neurooncol 2005;75(1): 63-69

2 Nayak L, Abrey LE, Iwamoto FM. Intracranial dural metastases. Cancer 2009;115(9):1947-1953

3 Pai SB, Krishna KN. Foramen magnum metastatic malignant melanoma. Neurol India 2003;51(1):79-80

4 Shimizu K, Takamiya Y, Furuhata S, Naritaka H, Kameya T. Cystic lesion at the foramen magnum disseminated from a pituitary adenoma-case report. Neurol Med Chir (Tokyo) 1999;39(8): 606-611
5 Margalit NS, Lesser JB, Singer M, Sen C. Lateral approach to anterolateral tumors at the foramen magnum: factors determining surgical procedure. Neurosurgery 2005;56(2, Suppl):324-336, discussion 324-336

6 Tremont-Lukats IW, Bobustuc G, Lagos GK, Lolas K, Kyritsis AP, Puduvalli VK. Brain metastasis from prostate carcinoma: The M. D. Anderson Cancer Center experience. Cancer 2003;98(2): 363-368

7 Greenberg HS, Deck MD, Vikram B, Chu FC, Posner JB. Metastasis to the base of the skull: clinical findings in 43 patients. Neurology 1981;31(5):530-537

8 Batson OV. The function of the vertebral veins and their role in the spread of metastases. Ann Surg 1940;112:138-149

9 Tomlin JM, Alleyne CH. Transdural metastasis from adenocarcinoma of the prostate mimicking subdural hematoma: case report. Surg Neurol 2002;58(5):329-331, discussion 331

10 Tagle P, Villanueva P, Torrealba G, Huete I. Intracranial metastasis or meningioma? An uncommon clinical diagnostic dilemma. Surg Neurol 2002;58(3-4):241-245

11 Fink LH. Metastasis of prostatic adenocarcinoma simulating a falx meningioma. Surg Neurol 1979;12(3):253-258

12 Nanda A, Vincent DA, Vannemreddy PSSV, Baskaya MK, Chanda A. Far-lateral approach to intradural lesions of the foramen magnum without resection of the occipital condyle. J Neurosurg 2002;96 (2):302-309

13 McDermott RS, Anderson PR, Greenberg RE, Milestone BN, Hudes GR. Cranial nerve deficits in patients with metastatic prostate carcinoma: clinical features and treatment outcomes. Cancer 2004;101(7):1639-1643

14 O'Sullivan JM, Norman AR, McNair H, Dearnaley DP. Cranial nerve palsies in metastatic prostate cancer-results of base of skull radiotherapy. Radiother Oncol 2004;70(1):87-90

15 Iwai Y, Yamanaka K, Ikeda H. Gamma Knife radiosurgery for skull base meningioma: long-term results of low-dose treatment. J Neurosurg 2008;109(5):804-810

16 Francel PC, Bhattacharjee S, Tompkins P. Skull base approaches and gamma knife radiosurgery for multimodality treatment of skull base tumors. J Neurosurg 2002;97(5, Suppl):674-676 